\title{
ARTICLE
}

\section{Shielding analyses for VISION beam line at SNS}

\author{
Irina Popova* and Franz X. Gallmeier \\ Oak Ridge National Laboratory, Spallation Neutron Source, PO Box 2008, MS6475, Oak Ridge, TN 37831-6471, U.S.A
}

Full-scale neutron and gamma transport analyses were performed to design shielding for the VISION beam line, the instrument cave, the beam stop, and the secondary shutter. The design goal is to achieve dose rates below $0.25 \mathrm{mrem} / \mathrm{h}$ at $30 \mathrm{~cm}$ from the shielding surfaces in generally occupiable areas. The secondary shutter analyses were performed with the discrete ordinate code DORT in addition to Monte Carlo analyses with the MCNPX code. Results from the shielding analyses are presented.

Keywords: high-energy accelerator; Monte Carlo, shielding design; dose rate distributions; neutron beam lines

\section{Introduction}

Spallation Neutron Source (SNS) in Oak Ridge, Tennessee, operates at 1 megawatt (MW) proton beam power incident on a mercury target with a proton beam energy of $1 \mathrm{GeV}$ and $60 \mathrm{~Hz}$ repetition rate. Most of the shielding work for the SNS is focused on the build-out of neutron beam lines and scattering instrument enclosures in order to provide a radiologically safe working environment during the beam operation. In this paper we discuss the shielding design analyses for the dual beam line 16, which consists of the operational beam line $16 \mathrm{~B}$, and the only partially built out beam line 16A. The vibrational spectrometer (VISION), at beam line $16 \mathrm{~B}$, is an inverted geometry instrument with a T0-chopper and one bandwidth chopper. The instrument is designed to use neutrons with energies in the range from $5 \mathrm{meV}$ to $500 \mathrm{meV}$ provided by the decoupled ambient water moderator. The moderator to sample distance is as short as possible - $16.0 \mathrm{~m}$. The design criteria for the shielding analyses is to achieve dose rates below $0.25 \mathrm{mrem} / \mathrm{h}$, at distance of $30 \mathrm{~cm}$ from the shielding surfaces in occupiable areas with a white beam (unobstructed beam, choppers assumed fixed in open position) hitting a thick steel sample. The thick steel sample is considered to represent the worst-case sample in terms of scattered radiation. Analyses are performed according to the guidelines for the SNS neutron beam line shielding calculations [1], which sets standards for the beam lines analyses.

\section{Beam line geometry}

The geometry model for the VISION beam line starts

\footnotetext{
*Corresponding author. Email: popovai@ornl.gov
}

at the target monolith and includes the beam line with the surrounding shielding, the instrument cave, and the two second floor rooms above the instrument cave. The instrument cave houses the beam stop. A vertical cross section of the complete general model is shown on Figure 1. The beam line shielding starts at $5.1 \mathrm{~m}$ from the moderator and it ends at $14.3 \mathrm{~m}$, where the instrument cave starts.

The beam line model includes the chopper cavity, which houses the T0 and bandwidth choppers, the secondary shutter, and the support structures. The T0 chopper model consists of two cylinders located one above the other. The lower cylinder is made from inconel and the upper cylinder (counter balance) is made from tungsten. In normal conditions, for the analyses both cylinders are located above the beam.

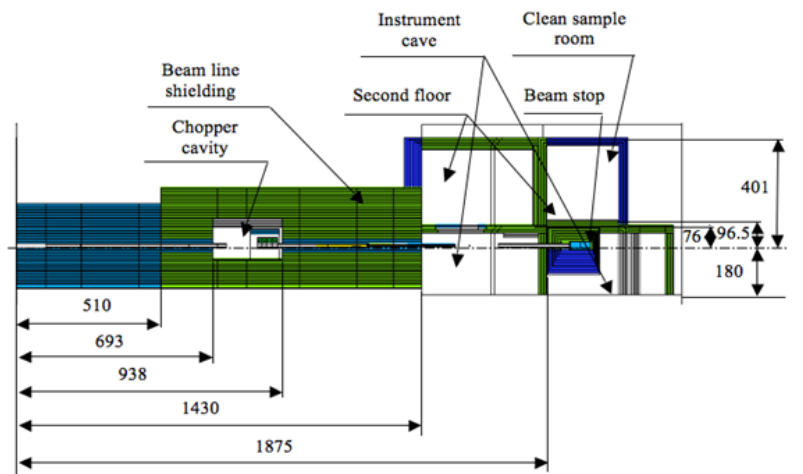

Figure 1. Vertical cross section from the moderator to the end of the instrument cave through the beam centerline. The dimensions are given in centimeters.

The dark blue color in the figures indicates regular concrete, the light blue color indicates steel, and the green color indicates high-density concrete.

The instrument cave starts at the end of the beam line shielding (14.3m), and its walls are built from the 
high-density concrete with a wall thickness of $30 \mathrm{~cm}$, Figure 2. The high-density concrete ceiling is 30 -cm-thick everywhere except under the clean room, where its thickness is increased to $50 \mathrm{~cm}$. The instrument cave houses a portion of the beam guide, the sample, the get-lost tube, and the beam stop. The sample is accessed through an opening in the ceiling, which is accessible from the second floor room located directly above. The access to the instrument cave is through a labyrinth, with a composite personnel door. The composite door is made from two 5-cm-thick steel layers sandwiching a layer of 5-cm-thick of polyethylene with $5 \mathrm{wt} \%$ boron loading.

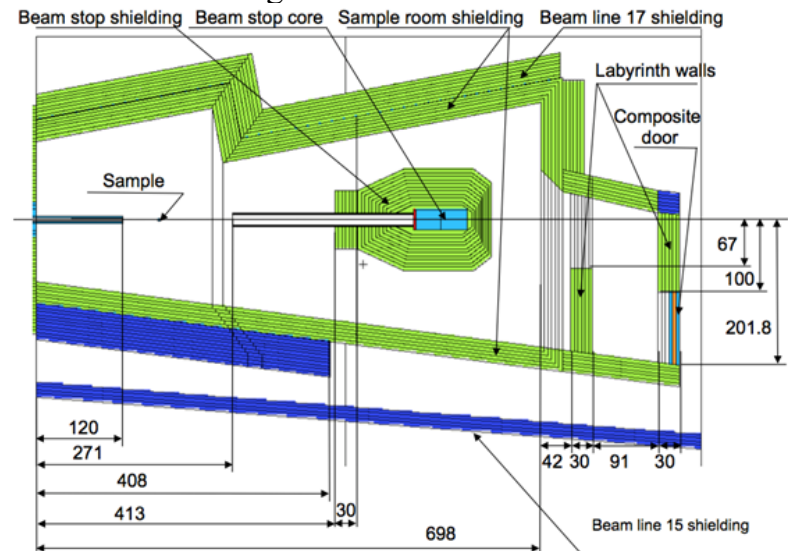

Figure 2. Vertical cross section from the moderator to the end of the instrument cave through the beam centerline. Dimensions are given in centimeters.

There are two rooms on the second floor above the instrument cave (Figure 1). One of those is the sample clean room, which will be occupied during the beam operation. The other second floor room provides access directly above the sample location for sample change outs, and will be closed by a composite door when the instrument receives the beam. The composite door is identical to the composite door for the labyrinth on the first floor. The second floor room walls are $30-\mathrm{cm}$-thick. However, the common wall between the sample access room and the sample clean room is $50-\mathrm{cm}$-thick and the ceiling above the second floor rooms is $45-\mathrm{cm}$-thick. The downstream wall and the beam line 17 side wall of the sample clean room, and the sample clean room ceiling are made from regular concrete. All other walls and ceiling are high-density concrete.

\section{Methods and codes}

Radiation transport calculations for shielding design and radiation protection analyses for SNS are performed mainly with the Monte Carlo code MCNPX version 2.5.0 [2].

However, there are tasks, where discrete ordinates methods are preferred at a much lower computational cost. These are tasks that combine streaming through a straight channel and deep penetration problems, such as analyses for the thick shielding (e.g., secondary shutters or temporary beam stops).
Effective dose rates are obtained by folding neutron and gamma fluxes with flux-to-dose conversion coefficients, which are taken from the standardized for SNS neutron and gamma flux-to-dose conversion factors libraries [3].

\subsection{Monte Carlo analyses}

The revised MCNPX 2.5.0 code version is used to transport particles through the guide. This version has a neutron mirror guide option, permitting the thermal and cold neutron transport in the neutron guides to be adequately modeled [4].

The neutron source was built by taking into account the neutron in-leakage into the beam line with an opening of $100 \mathrm{~mm} \times 120 \mathrm{~mm}$ starting at about $1 \mathrm{~m}$ from the moderator face. The source consists of two angular segments, the first segment is a cone from 0 to 1 degree, and the second segment is a cone from 1 to 2 degrees with regard to the nominal direction of the neutron beam line. Each of the two angular segments has its own energy spectrum. In order to make calculations more efficient and to apply source biasing, the initial two angular segments were transformed into a four-angular-segment source by subdividing the first angular segment into 3-angular segments. This segmentation allows the transport of a higher number of neutron histories (with lower weight) from the smallest angle, which mostly illuminates the end of the guide.

Geometry splitting was used to keep the same particle population in the layers towards the outside of the shielding for the beam line shielding and for the sample cave shielding analyses.

For scoring the neutron and gamma dose rates, two types of tallies were used: mesh and surface tallies. The mesh-tallies were defined in the vertical and horizontal planes positioned at the beam centerline, above the beam, or aside of the beam, with mesh sizes of $5 \mathrm{~cm} \times 5 \mathrm{~cm}$ in the plane and up to $10 \mathrm{~cm}$ in the out of plane direction. The surface tallies were set on corresponding surfaces in key locations.

\subsection{Discrete ordinates analyses}

Discrete ordinate analyses are performed for estimating the radiation attenuation of the secondary shutter and temporary beam stop for the neighboring beam line 16A. DORT, a two-dimensional discrete ordinates code [5] was used. In order to mitigate ray effects, the auxiliary code, GRTUNCL [6], was applied to produce a first collision source, which is distributed throughout the entire geometry. For source terms, a spectrum corresponding to a 1 degree MCNPX source was used and normalized, so that the in-beam dose rate incident to the secondary shutter or to the temporary beam stop is the same as in the MCNPX calculation. A two-dimensional simplified model in $(\mathrm{r}, \mathrm{z})$ geometry (cylindrical) was developed for the DORT analysis. In order to give a refined description of the propagation of the radiation into and through the openings the highly biased in forward direction quadrature set is used, which 
includes 315 directions. The maximum order of scattering used in the problem is P5.

\section{Results}

Beam line shielding analyses are logically divided into two parts: analysis of the incident beam line and analysis of the instrument enclosure, including the neutron beam stop.

\subsection{Incident beam line shielding}

Beam line shielding analyses are performed for different operational scenarios:

- White beam (all choppers open or removed);

- T0 chopper in closed position, secondary shutter in open position;

- Secondary shutter in closed position, T0 chopper in open position.

These analyses include the secondary shutter design.

\subsubsection{Beam line shielding}

Figure 3 shows the dose rate map in vertical cross section through the beam line, when the chopper and secondary shutter are in the open position. Dose rates at the shielding surface are below $0.25 \mathrm{mrem} / \mathrm{hr}$. Analyses for the scenarios, when the chopper or shutter obstruct the beam, resulted in surface dose rates below the design goal of $0.25 \mathrm{mrem} / \mathrm{h}$.

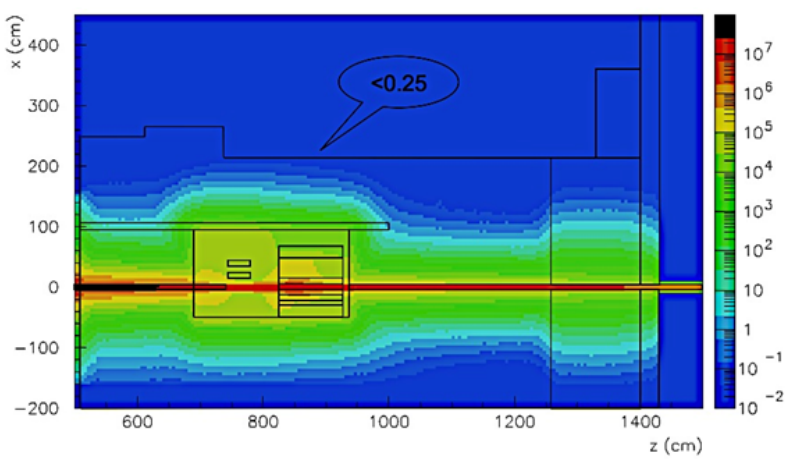

Figure 3. Dose rate map in vertical cross section through the beam line, chopper and secondary shutter are in open position, $\mathrm{mrem} / \mathrm{h}$.

After beam line shielding was installed, a gap about 2.54-cm-wide in the shielding between the beam lines 17 and $16 \mathrm{~B}$ was identified and was due to imperfection of the block dimensions. The worst case from the point of view of radiation streaming through the gap will be when the chopper or the shutter is in the beam. Including the gap into the model indicates dose rates as high as $95 \mathrm{mrem} / \mathrm{hr}$ on the top of the shielding. In order to mitigate the elevated dose rate, a $30 \mathrm{~cm}$ thick key block of high-density concrete is placed above the gap, and the gap clearance is restricted to $0.63 \mathrm{~cm}$.

\subsubsection{Secondary shutter}

A secondary shutter is required due to beam line $16 \mathrm{~B}$ being part of a dual beam line. In order to allow access to the beam line 16B instrument cave while beam line $16 \mathrm{~A}$ is open, beam line $16 \mathrm{~B}$ utilizes a secondary shutter.

A first set of analyses was performed with MCNPX to calculate the required secondary shutter length with tungsten as material. However, the results did not converge well enough, and DORT code was used as a second approach. Only $30 \mathrm{~cm}$ in radial direction was modeled in the discrete ordinate analyses (see Figure 4), because any further geometry would not impact the results for shutter length calculation. The beam pipe was modeled as a voided set of cylinders with gradually decreasing radii towards the sample. The total dose rates (gammas and neutrons) inside the beam pipe calculated with MCNPX and DORT are presented in the Figure 5. Results from MCNPX calculations are shown with a red line for a shutter thickness of $70 \mathrm{~cm}$. Results from the DORT calculations are shown for shutter lengths of 90 $\mathrm{cm}$ and $80 \mathrm{~cm}$ and for the open shutter position. MCNPX gives an underestimated dose rate at the end of the guide position because of the poor sampling of high-energy neutrons at solid angle elements close to the beam line axis penetrating the shutter. At the end of the beam guide, the dose rate for the $70 \mathrm{~cm}$ tungsten shutter is $0.1 \mathrm{mrem} / \mathrm{h}$. DORT requires shutter thicknesses of 90 $\mathrm{cm}$ and $80 \mathrm{~cm}$ to obtain 0.1 and $0.4 \mathrm{mrem} / \mathrm{hr}$, respectively. A secondary shutter thickness of $80 \mathrm{~cm}$ was selected.

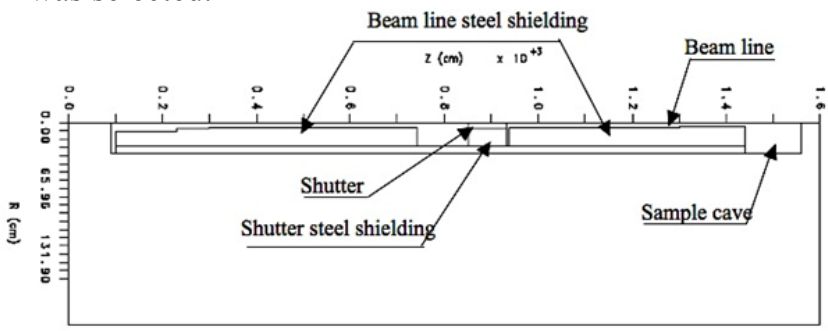

Figure 4. VISION beam line DORT model.

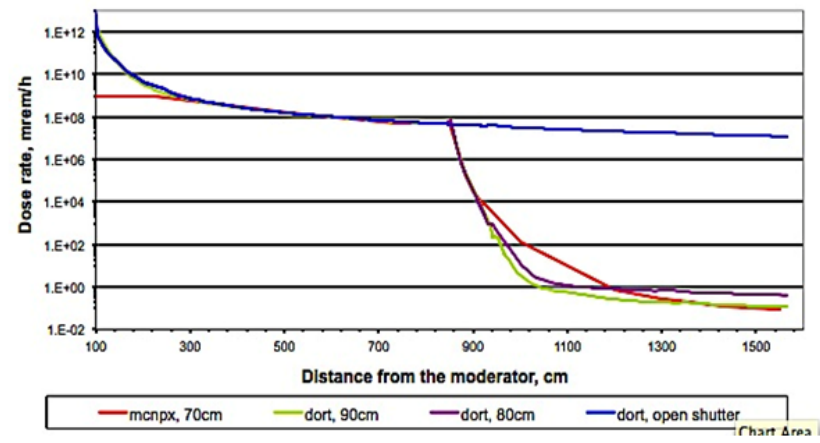

Figure 5. Total dose rates calculated MCNPX and DORT for various shutter lengths.

\subsection{Instrument enclosure and beam stop shielding}

The instrument enclosure shielding analyses includes two separate analyses:

- Beam stop shielding design;

- Instrument enclosure shielding design.

\subsubsection{Beam stop design}

The VISION beam stop is located inside the enclosure as shown in Figures 1 and 2. The design 
criteria was to achive dose rate below $0.25 \mathrm{mrem} / \mathrm{h}$ at 30 $\mathrm{cm}$ from the instrument enclosure surface. Shielding analyses are performed for the "white" beam running without interception by a sample into the beam stop.

The beam stop consists of core and shielding parts. The beam stop core is a 72-cm-long steel cylinder with diameter of $28 \mathrm{~cm}$ and with a $2.54 \mathrm{~cm}$ thick boron carbide disk at the front face. The beam stop core is surrounded by high-density concrete all the way to the ceiling and $56-\mathrm{cm}$-thick on the sides. The whole beam stop assembly is sitting on a regular concrete base. Figures 6 and 7 show dose rates in the vicinity of the beam stop. The beam stop shielding meets the criteria of the shielding design with exception of the area between the instrument enclosure and BL15, mitigation is discussed in the next section.

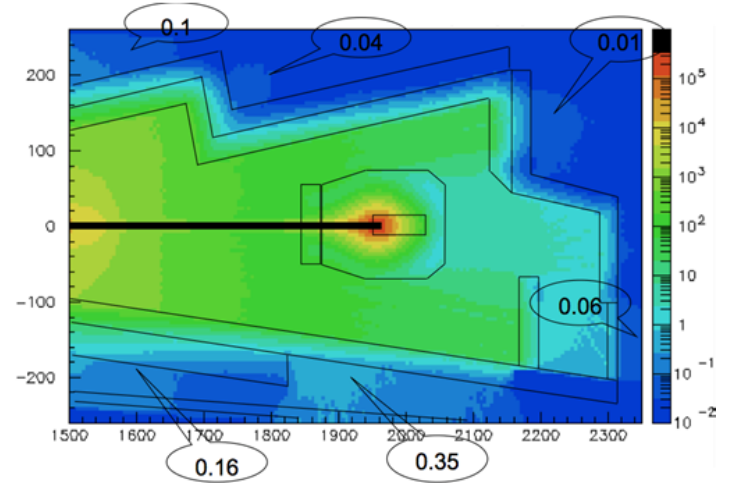

Figure 6. Dose rate map in horizontal cross section through the beam line, $\mathrm{mrem} / \mathrm{h}$.

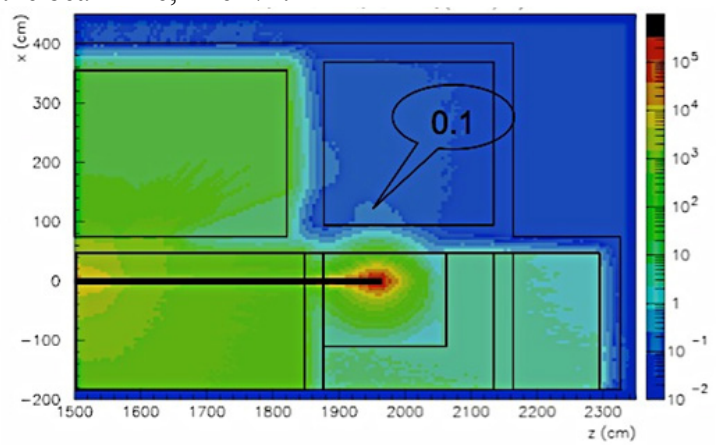

Figure 7. Dose rate map in vertical cross section through the beam line, $\mathrm{mrem} / \mathrm{h}$.

\subsubsection{Instrument enclosure shielding design}

Shielding for the instrument cave was designed in a couple of calculation iterations. Shielding design for the instrument cave includes:

- Design of the shielding labyrinth to access the sample cave;

- Design of two composite doors, one door for the cave and the other for the second floor room;

- Wall and ceiling thicknesses for the first floor;

- Wall and ceiling thicknesses for rooms on the second floor.

Sample cave shielding analyses were performed for the "white", un-chopped beam incident on the worst-case sample in terms of neutron scattering and gamma production, a steel cylinder of $4 \mathrm{~cm}$ in diameter and $6 \mathrm{~cm}$ length.
The design for the labyrinth, doors and the wall thicknesses are as described in Section 2. Figure 8 shows a dose rate map in the horizontal cross section inside and outside of the instrument enclosure.

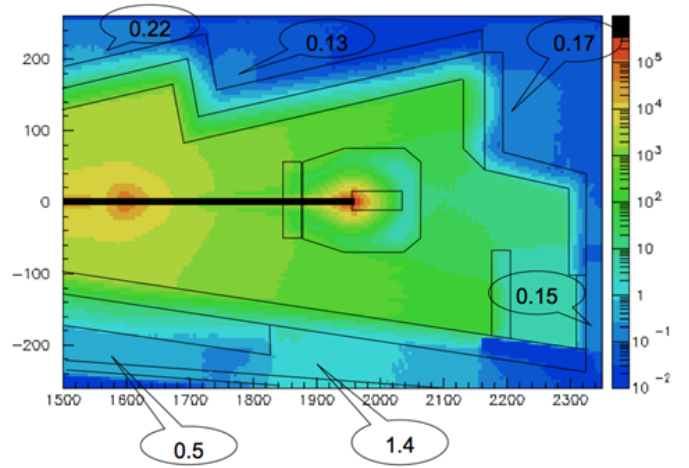

Figure 8. Dose rate map in vertical cross section through the beam line, $\mathrm{mrem} / \mathrm{h}$.

A slightly elevated dose rate, up to $1.4 \mathrm{mrem} / \mathrm{h}$, in the area between the instrument enclosure and the BL15 structures, is mitigated by restricting acces to the area. Dose rates elsewhere are within design criteria.

\section{Conclusion}

Full-scale analyses were performed to design shielding around VISION beam line, instrument shielding enclosure, beam stop and secondary shutter. Meanwhile the beam line has been commissioned and operates in the present time with the designed shielding. Fault study measurements confirmed the adequacy of the shielding design.

\section{Acknowledgements}

Work supported by the Division of Materials Science, U.S. Department of Energy, contract number DE-AC0596OR22464 with UT-Battelle Corporation for ORNL.

\section{References}

[1] I. Popova, Guidelines for Shielding Calculations for SNS Neutron Beam Lines, ORNL, SNS 106100200-WI0001-R01, August 2012.

[2] Pelowitz D. B., editor, MCNPX User's Manual Version 2.5.0, LA-CP-05-0369, Los Alamos National Laboratory, Los Alamos, April 2005.

[3] I. Popova, Flux to Dose Conversion Factors, SNS-NFDD-NSD-TR-0001, R00, October 2009.

[4] F.X. Gallmeier, M. Wohlmuther, U. Filges, D. Kiselev and G. Muhrer, Implementation of neutron mirror modeling capability into MCNPX and its demonstration in first applications, Nucl. Tech. 168 (2009), pp. 768-772.

[5] W.A. Rhoades and R.L. Childs, The DORT two-dimensional discrete ordinates transport code, Nucl. Sci. \& Engr. 99 (May 1988), pp. 88-89.

[6] R.L. Childs, GRTUNCL, First Collision Source Program, ORNL Informal Notes (1962). 